\title{
Microbes in the Datura rootzone contribute to an antioxidant support system of flavonoids and other aromatic compounds
}

\author{
Savanah Senn ${ }^{1,2 *}$, Kelly Pangell ${ }^{1}$ and Adrianna L. Bowerman ${ }^{1}$ \\ 1 Agriculture Sciences Department, Los Angeles Pierce College, Woodland Hills, CA \\ 2 Environmental Sciences Graduate Program, Oregon State University, Corvallis, Oregon \\ * Correspondence: stclais@piercecollege.edu
}

\begin{abstract}
The purpose of this paper is to elucidate the roles that microbes may be playing in the rootzone of the medicinal plant Datura inoxia. We hypothesized that rhizospheric and endophytic microbes would be found that were capable of performing the same secondary metabolic functions of the plant rootzone they inhabited. We also hypothesized that the microbial functions would be cooperative with and supportive to plant secondary metabolite production, for example, by providing precursors to important plant bioactive molecules. The methods employed were microbial barcoding, tests of essential oils against antibiotic resistant bacteria and other soil bacterial isolates, $16 \mathrm{~S}$ Next Generation Sequencing (NGS) metabarcoding, and Whole Genome Shotgun (WGS) taxonomic and functional. A few of the main bacterial genera of interest that were discovered in the Datura root microbiome were Flavobacterium, Chitinophaga, Pseudomonas, Streptomyces, Rhizobium, and Bacillus. In the context of known interactions, and current results, plants and microbes influence the flavonoid biosynthetic pathways of one other, in terms of the regulation of the phenylpropanoid pathway. This is important because these compounds are phyto-protective antioxidants and are precursors to many aromatic bioactive compounds that are relevant to human health. There was strong evidence to support the notion that synergistic production of plant derived secondary metabolites by microbes occurred, as well as the ability for the compounds to enter plant cells. There are possible biopharmaceutical and agricultural applications of the natural interplay that was discovered during this study of the Datura inoxia rhizosphere.
\end{abstract}

Keywords: Weed science, Plant-microbe interactions, Medicinal plants, Shotgun metagenomics, Soil metabarcoding

\section{Introduction}

The Significance of the Plant

The Datura plant genus produces several important cyclic compounds, most notably the tropane alkaloids. Datura produces alkaloids for anti-herbivory including scopolamine and atropine. Both of these alkaloids have medicinal uses, however at high doses they are toxic and can lead to hallucinations, or even death. Atropine is an antidote for nerve gas because it blocks acetylcholine activity. Atropine can also be used to treat asthma and low heartrate. Scopolamine is used for prevention of nausea and motion sickness.

Datura has analgesic properties [1]. Traditionally, the flowers have been worn around the ears to treat earache. Furthermore, the plant contains antioxidants such as flavonoids, and phenols. Quercetin is a flavanol with anticancer properties that has free radical scavenging activity. Other compounds may include scopoletin [2], which is a fluorescent coumarin that has antifungal properties against antibiotic resistant strains of Candida [3]. The plant does not produce nicotine like some other members of Solanaceae.

This plant is sometimes referred to as an entheogen due to its use by native people in the United States such as the Chumash. Datura has been used for rituals and ceremonies in 
Hindu culture [4]. Since the flower is valued for its aesthetic properties, it is also grown as an ornamental plant and there are fertilizers that are developed specifically to promote flowering. It is also occasionally used as a hallucinogen in a non-spiritual setting.

Datura inoxia has grey-green, flat, simple foul-smelling leaves that typically lack toothed margins. The leaves resemble wild tobacco leaves and may contain ornithine and putrescine, which attracts predatory birds. The leaves are slightly downy. It is most readily identified by the white, sweet-smelling inflorescence, which blooms in the evening and attracts pollinators such as Sphinx moths. The blooms resemble Brugmansia. It is an outcrossing plant, like many of the Solanaceae. The flower exhibits heterostyly; the female part sticks out of the flower and may be receptive before the pollen is dehiscent, contributing to heterozygosity. The round seed pods are spiny and lignified and can be dehiscent. The seeds pods have a round fleshy placenta within, which is covered by numerous flat seeds, resembling a bell pepper on the inside. The seeds are highly heterozygous and spontaneous mutations are common in tissue culture [5].

A close relative of Datura inoxia, D. wrightii was recently recognized in rock art paintings by the native Chumash at Pinwheel Cave [6]. The nature of the altered state of consciousness theory was recently challenged by the discovery of rock art from Pinwheel Cave in Kern County, CA. Anthropologists had theorized that rock art paintings were created in an altered state of consciousness. Although anthropologists long believed that the rock art paintings depicted a vision or hallucination, the recent discoveries paint a different picture.

The cave art also depicts an anthropomorphic sphinx moth, which is known to pollinate the plant. Interestingly, sphinx moths have often been seen flying erratically after visiting Datura flowers (Ibid). Rather than depicting a hallucination, the rock art depicts the sacrament itself and reflects the natural cycles of flowering and pollination that are essential to this dynamic outcrossing plant. The cave was stuffed with at least 15 quids of Datura flowers that had been chewed by groups of up to 10 people. Groups also used the cave to prepare for hunting expeditions for several generations, and as a seasonal food preparation place. Toloache is a native drink preparation that is well-known as part of an adolescent initiation ceremony; these quids were chewed like tobacco and then stuffed into the ceiling of the cave. Beyond adolescence, Datura was used by native people to gain personal power, including for doctoring and hunting trips, and to treat ailments.

\section{The Significance of the Plant Microbiome}

Recently, a few studies have elucidated the interaction between plants and microbes during secondary metabolite production. It is known that in some species, such as Crotalaria, nodulation is required for alkaloid production [7]. Functional analysis of soil metagenomic data has expanded as a field, and is used as a way to consider how plants are influencing the microbiome [8]. The microbiome is equally important in understanding plant secondary metabolism.

Shotgun metagenomics approaches in applied soil science provide the advantage of amplifying sequences from throughout the genome, including coding regions, yielding a high sequencing depth but at a higher cost. 16S metabarcoding is used as a diagnostic tool in clinical sciences and plant ecology, and its forté is identification of bacteria and archaea. However, metagenomics and metabarcoding are culture independent, meaning that no viable accessions are maintained. For that reason, microbial barcoding of streak plates isolated from the soil is also a useful tool that provides material for later wet lab experiments involving plant inoculation or antimicrobial trials, and helps to validate NGS results with tangible materials. 


\section{Flavobacterium and Chitinophaga}

According to Sang et al 2012, Flavobacterium johnsoniae produced 2,4-di-tert-butylphenol which had biocontrol activity against Phytophthora in Capsicum [9]. This references one of a few studies in Solanaceae where Flavobacterium has been shown to suppress disease. Another example is Carrion et al 2019, where Rhizoctonia solani was suppressed by the endophytic root microbiome [10]. Some of the most important natural products involved were phenazines, polyketides, siderophores, and chitinases. Aryl polyenes, which are structurally similar to carotenoids, were enriched by Flavobacteriaceae; terpenes and resorcinol were enriched by Chitinophaga. Similarly, in 2020 Lucke et al reported that as biocontrol agents, the secretion of secondary metabolites triggered the induction of systemic resistance in plants [11]. In particular, they pointed out the importance of the chitinase from Flavobacterium and the Polyketide Synthase (PKS) gene cluster from Chitinophaga that is essential for disease suppression.

\section{Mycobacteriaceae}

Del Barrio-Duque et al 2019 found that 17/19 Mycobacteriaceae strains tested had fungal growth-promoting properties on Serendipita indica in vitro and in tomato; they were plant growth-promoting and helped to alleviate symptoms of Rhizoctonia solani [12]. In this research, Mycolibacterium was in a consortium with Rhizobium and Paenibacillus; in some instances, the isolations worked better than combinations of inoculants. Surprisingly, Burkholderia had a negative impact in vitro on $S$. indica fungal growth, although it has been shown to be plant growth promoting. An interesting review by Morris et al 2019 highlights the fact that benzylisoqunioline alkaloid methyltransferases are functionally similar to the cyclopropane methyltransferase from Mycobacterium tuberculosis [13]. Mycobacterium cyclopropanation could contribute to tropane alkaloid biosynthesis in Datura, or provide an intermediate toward the same.

\section{Rhizobium and Streptomyces sp.}

Banuelos-Vazquez reported that endophytes can receive nodulation genes, and in some cases, the ability to fix nitrogen, from Rhizobium etli. Plasmids are transferred to other endophytes in plant roots [14]. Another interesting example is from Kado and Kelly 2006, where they reported a successful protocol for transforming Streptomyces with Agrobacterium [46]. Lucke 2020 also noted the root nodulating capabilities and transfer of plasmids by R. etli and A. tumefaciens, and their ability to induce plant defenses [11].

Furthermore, the plant growth-promoting activities of endophytes may include production of plant growth regulators such as Indole Acetic Acid (IAA), and siderophore production for iron acquisition. Streptomyces spp. within Bruguiera gymnorhiza and Boesenbergia rotunda increased flavonoids and cyclopeptides with anti-HIV and anticancer activities [15]. Endophytic bacteria, as well as fungi, can produce paclitaxel [16]. Within Taxus baccata, Streptomyces produced 0.01-0.02 ng/mL, and Bacillus subtilis produced 1-25 $\mathrm{ng} / \mathrm{mL}[16]$.

Indeed, as $\mathrm{Wu}$ et al pointed out, the relationship between medicinal plants and endophytes is a long-term, symbiotic relationship [15]. Endophytes can strongly regulate the synthesis of secondary metabolites in plants. For example, for abiotic stress tolerance, in Pteris vittata, Agrobacterium and Bacillus spp. reduced arsenate to arsenite.

\section{Pseudomonas and Bacillus sp.}

Volatile organic compounds can also help bacteria that are physically separated communicate with one another. Endophytic bacteria can protect plants by quenching quorum sensing molecules. For example, Pseudomonas aeruginosa could degrade 3- 
hydroxy palmitic acid methyl ester, which is a quorum sensing molecule of Rhizoctonia solanacearum. The inoculation of eggplant with $P$. aeruginosa reduced bacterial wilt caused by R. solanacearum [15]. In tomato, Bacillus aureus and Serratia nematodiphilia were applied to seeds along with Ralstonia syzigii sub-infection. The treatment under inoculation had increased jasmonic acid concentration in leaves and roots until 12 days post-infection [17].

Antioxidant enzymes are activated by endophytes, such as phenylalanine ammonia lyase, tyrosine ammonia lyase, and polyphenol oxidase (PPO) [17]. Rhizobacterial root colonization significantly impacted phenolic compounds, terpenes, and essential oils in plants. Pseudomonas putida colonization root colonization altered benzoxaninone levels three days after inoculation. Colonization by Rhizobium changed the levels of phenolics, flavonoids, and anthocyanins, in blackberry, which was associated with delayed fungal postharvest growth [17].

In terms of the accumulation of plant secondary metabolites, production of plant sesquiterpenoids was enhanced in Atractyloides macrocephala by Pseudomonas fluorescens. Essential oil production was also increased [17]. According to Sang et al 2012, endophytes can increase resistance to insects, while the plants provide nutrition and protection [9]. In Nethapodytes fortida and Apodytes dimidiata, two grasses, Fusarium solanii fungi were found to produce campothecin. This quinoline compound was previously taken from the roots of Nethapodytes directly.

Purpose of the Study

The purpose of this paper is to elucidate the roles that microbes may play in the rootzone of the medicinal plant Datura inoxia. We hypothesize that we will find rhizospheric and endophytic microbes performing the same secondary metabolic functions of the plant rootzone they inhabit. We also hypothesize that the microbial functions will be cooperative with and supportive to plant secondary metabolite production, for example, by providing precursors to important plant bioactive molecules.

\section{Materials and Methods}

Soil samples were collected in triplicate from the field and arboretum at Los Angeles Pierce College. Replicated samples were taken from plant rootzones in the top $3 \mathrm{~cm}$ within a 1-foot radius of the plant subjects including Alnus rhombifolia, Datura inoxia, Ethiopian Eragrostis tef (Teff) grass, Opuntia cactus rootzone, and fallow conditions. They were stored at $-20^{\circ} \mathrm{C}$ and DNA was extracted using the Qiagen Power Soil DNA kit and sent to Cold Spring Harbor Laboratory for $16 \mathrm{~S}$ amplification, library preparation, and pooled $16 S$ amplicon NGS on the Illumina MiSeq platform. Soil from the same sampling event was sent to Beijing Genomics Institute America, San Jose for logistics and processing, for the replicated Datura, Alnus, and fallow rhizospheric soil samples.

According to the USDA NRCS Web Soil Survey historic data, the fields related to the Datura, Alnus, and fallow samples are all classified identically as Cropley-Urban Land Complex 0-2\% slopes, and the reported measurements for $\mathrm{pH}$, texture, cation exchange capacity (CEC), and percent organic matter are identical, as shown in Table 1 [18].

Table 1. Soil Physical and Chemical Properties of the Fields sampled based on Historical USDA data.

\begin{tabular}{l|l|l|l|l|l|l|}
\hline Map Unit Name & pH & CEC & \%Sand & \%Silt & \%Clay & \%OM \\
\hline $\begin{array}{l}\text { Cropley-Urban land complex, 0 to } \\
2 \%\end{array}$ & 7.9 & 37.5 & 22.1 & 27.9 & 50 & 1.5 \\
\hline
\end{tabular}


Whole genome shotgun sequencing, DNA extraction, and library preparation was subsequently performed by Beijing Genomics Institute on the DNBseq platform. Preliminary trim and QC were carried out using SOAPnuke [19]. MG-RAST was used to generate functional and taxonomic profiles [20]. Taxonomic identification was performed using RefSeq and functional profiles were built from Subsystems identifiers. In order to determine which taxa were differentially abundant between categories, DESeq2 was used [21]. STAMP was used for functional analysis [22].

For soil metabarcoding and 16S barcoding analyses, DNA Subway was used [23]. For soil metabarcoding, DNA extraction was performed with the Qiagen PowerSoil kit. Library preparation followed the Earth Microbiome Project 16S Illumina Amplicon protocol [24, 25]. Sequencing was performed at Cold Spring Harbor Laboratories. The Greengenes identifier (utilizing 515F/806R primers) was used.

Bacterial isolations were also generated for the Alnus, Datura, and fallow rhizosphere samples in order to provide material for future in vivo plant experiments according to the Soil Science protocols published by St. Clair et al [26]. Isolates from the soil solutions at $10^{\wedge}-3,10^{\wedge}-4$, and $10^{\wedge}-5$ dilutions were plated and grown for three days on various media, summarized in Table 2. Selected isolates were streaked on plates of the same media and colony PCR was performed after three days of isolated growth. For bacterial isolations, DNA was extracted by boiling colonies with chelex beads for 10 minutes, followed by $16 \mathrm{~S}$ rRNA amplification. Amplicon sizes were $>500 \mathrm{bp}$. Sanger sequencing for bacterial isolates was performed by Genewiz. QC was performed and consensus sequences were generated in the Cyverse DNA Subway Blue Line. The Phylip Maximum Likelihood tree was generated in Cyverse. Bacterial putative identifications for the isolates were generated using the EZBioCloud 16S-based ID application [27].

Lemon balm (Melissa officianalis) and Tea tree (Maleleuca alternifolia) essential oils are well-characterized. Vegetative plant material was harvested from and air dried at room temperature for one week. The samples were handled separately. Soxhlet extraction was carried out for three cycles using reagent ethanol as the solvent. The extracts were subsequently dewaxed by running them through a Buchner funnel and filter paper.

Bacteria were cultured in $1.5 \mathrm{~mL}$ tubes of nutrient broth for 48 hours at room temperature. Separate sterile glass tubes filled with $2 \mathrm{~mL}$ of nutrient broth were inoculated with $100 \mathrm{uL}$ of each bacterial broth culture. The glass tubes were inoculated with $20 \mathrm{uL}$ of either lemon balm oil or tea tree oil from the soxhlet extractions. The clarity of the solution, which was a proxy for bacterial colonization levels, was measured as \% transmission at $600 \mathrm{~nm}$. Transmittance at $600 \mathrm{~nm}$ was measured using a spectrophotometer after culturing at room temperature overnight. The data was analyzed using $\mathrm{R}$.

\section{Results}

Soil samples WGS and MG-RAST taxonomic and functional assignments

The sequence count ranged from 4,608,913 to 34,551,930 sequences. Interestingly, and in contrast to the DNA Subway Purple line results, each category between the Datura rootzone, Fallow field near the compost pile, and the Alder rootzone soil had a sample in the top 3 best performers for read counts, but the Fallow field tended to have lower read counts overall, which is more of what one would expect considering no plants were growing there.

Figure 1. MG-RAST classification of the NCBI RefSeq identification of the soil organisms at the family level, for a representative sample of Datura rootzone soil. 

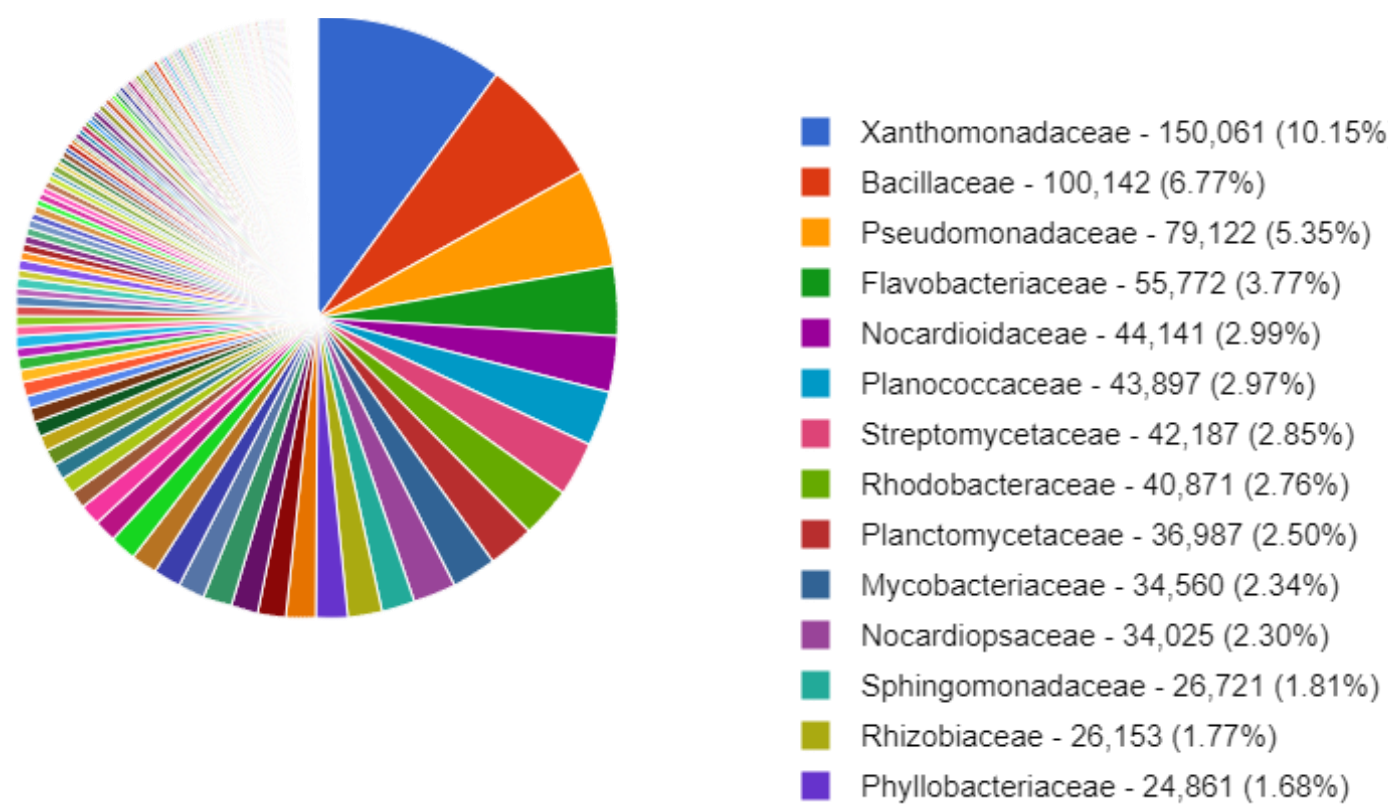

In the Datura rootzone, some of the most abundant genera were Pedobacter and Flavobacterium. Flavobacterium johnsoniae is well-known for its tyrosine ammonia lyase gene, which has been cloned and used in genetic transformations to optimize production of aromatic compounds of pharmaceutical value in microbial expression systems. Some potential applications are production of tropane alkaloids and p-coumaric acid [28, 29].

Figure 2. Relative abundance of the top genera in a representative sample of $\mathrm{Da}$ tura rootzone soil from the Pierce Farm in Los Angeles, California.

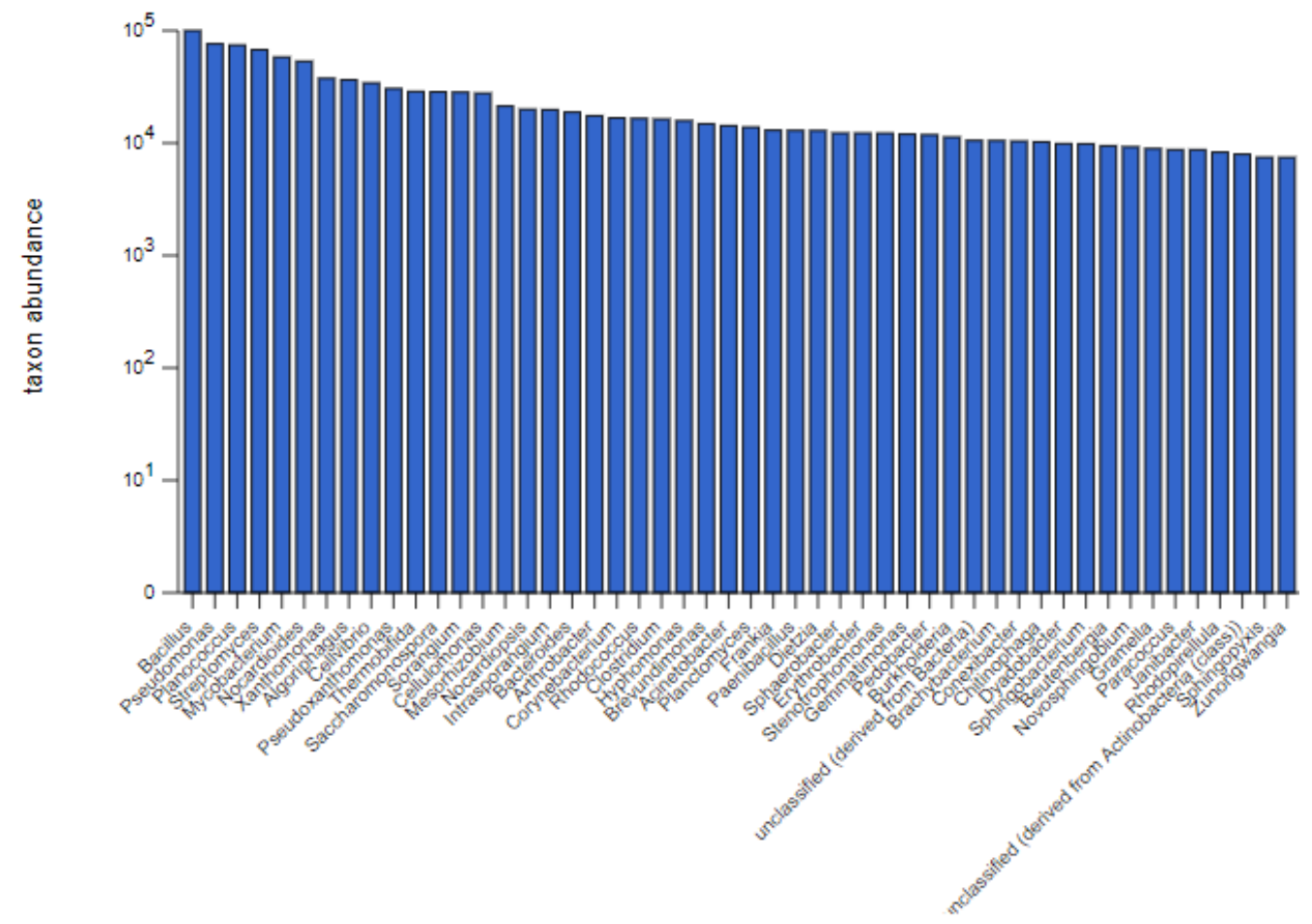

Functional Analysis in STAMP

Using a cutoff of alpha $=0.05$, the following were significantly higher in the Daturaassociated soil samples: Alpha-xylosidase $(\mathrm{p}<0.017)$, chalcone synthase $(\mathrm{p}<0.002)$ (Figure $3)$, putrescine utilization $(\mathrm{p}=0.05)$, Aromatic amino acid transport protein $\operatorname{AroP}(\mathrm{p}<0.003)$, polyols $A B C$ transporter permease component $(p<0.003)$, Quinate permease $(p=0.002)$, ubiquinone biosynthesis enzyme COQ $7\left(\mathrm{p}<7^{*} 10^{\wedge}-3\right)$, Heavy metal sensor histidine kinase $\left(\mathrm{p}<6.4^{*} 10^{\wedge}-3\right)$, flavin reductase family $(\mathrm{p}=0.022)$, para-hydroxybenzoate- polyprenyl transferase $(p<0.004)$, Siderophore achromobactin ABC transporter $(p<0.0021)$, NADPH quinone oxidoreductase $2(\mathrm{p}<0.0021)$, vanillate $\mathrm{O}$-demthylase oxygenase subunit $(\mathrm{p}<0.0014)$, 
Phenylacetic acid degradation protein PaaN $(\mathrm{p}<0.0013)$, clavulanic acid biosynthesis $(\mathrm{p}=0.027)$, cobalamin synthesis $(\mathrm{p}<0.005)$, and MAP kinase pathways $(\mathrm{p}<0.0045)$.

Figure 3. Differential abundance of chalcone synthase genes was detected in the Datura rootzone based on STAMP analysis of the MG-RAST generated Subsystems metagenomic functional profile.

Box plot

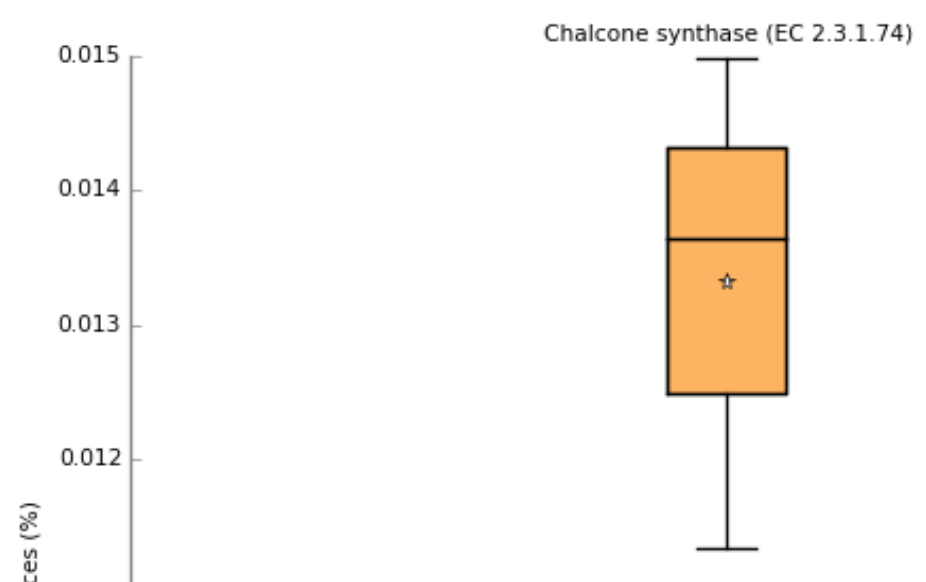

$p=1.94 \mathrm{e}-3$

0.01

0.009

0.008

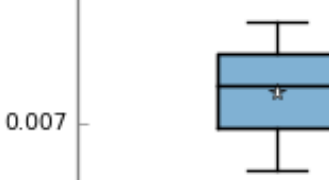

Alnus

Datura

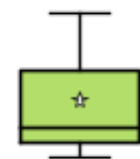

0.006

Fallow

On the other hand, the $\mathrm{Na}+$ translocating NADH-quinone reductase subunit was differentially abundant in Marquis $C\left(p<7^{*} 10^{\wedge}-4\right)$, the fallow field near the compost pile. Gram positive cell wall components were differentially abundant in Marquis $C(p<0.008)$, although gram negative cell wall components were equally likely to be present in all samples $(p=0.244)$. The CRISPR associated protein TM1812 was enriched in the fallow field near the compost pile $(\mathrm{p}<0.0014)$. Phenlypropanoid compound degradation functional genes were most abundant in the Alnus samples $\left(\mathrm{p}<6.4^{*} 10^{\wedge}-5\right)$. Tocopherol biosynthesis genes were also differentially abundant in the Alnus rhizospere-associated soil $(\mathrm{p}<0.004)$.

There was enrichment of cell wall degradation and depletion of gram-positive bacteria, stress response, siderophores, catabolism of tyrosine, enrichment for aromatic compounds and permeases, production and reduction of flavonoids, antibiotics and cobalamin, and degradation of oxidized products in the Datura rootzone. The presence of differentially abundant permease components for essential oils and quinates suggests that there is a secretion pathway that would allow these compounds to enter plant roots and contribute to flavonoid, alkaloid and terpenoid biosynthesis in plant hosts.

Purple Line analysis and comparison

Demultiplexed sequence counts for the DNA Subway Purple Line paired end data set ranged from 5,472 to 3.63 million. The lowest number of reads were for one of the 
Datura rootzone soil samples and a tilled fallow sample nearby in Marquis C. The highest number of reads were from Field 17, a fallow field tilled with cow patties and Marquis $\mathrm{C}$ near the compost pile. Indeed, in Field 17 also had a trend toward the highest alpha diversity, followed by Marquis C. There appeared to be more within group variation reflected by the $16 \mathrm{~S}$ workflow compared to WGS.

Enrichment of Pedobacter matched up with MG-RAST results, and with Mycobacterium which may be related to alkaloid biosynthesis. Pedobacter represented $>32 \%$ of the reads for one of the Datura rootzone samples. Blastococcus sequences were abundant in all three Datura rootzone soil samples. Streptomyces, Rubrobacter and Mycobacterium were abundant in one of the Datura samples, but were not detected in the other two. There appeared to be a similar drought tolerant entourage of bacteria associated with Datura, Teffgrass, and the cactus-associated soil samples, based on the Principal Coordinates Analysis (DNA Subway Purple Line Public project 7102).

Some unexpected results were detected by the isolation of bacterial colonies and analysis on the Blue Line. Four Chryseobacterium accessions were isolated from the soil that did not show up on $16 \mathrm{~S}$ amplicon sequencing for the same soil samples (Figure 4). However, the Chryseobacterium genus was detected with DNBseq WGS (see Table 4).

Table 2 Bacterial Strains Isolated and Culture Conditions

\begin{tabular}{|c|c|c|c|c|c|c|}
\hline $\begin{array}{l}\text { Sample } \\
\text { No. }\end{array}$ & Field & $\begin{array}{l}\text { Colony } \\
\text { Color }\end{array}$ & Morphology & $\begin{array}{l}\text { Antibiotic } \\
\text { Used }\end{array}$ & Media & $\begin{array}{l}\text { Absorbed } \\
\text { CR }\end{array}$ \\
\hline 1 & Marquis C & $\begin{array}{l}\text { Transl } \\
\text { yellow }\end{array}$ & Unkown & NA & $\begin{array}{l}\text { Nutrient } \\
\text { agar }\end{array}$ & NA \\
\hline 2 & Marquis D & $\begin{array}{l}\text { White/ } \\
\tan \end{array}$ & Unkown & Penicillin & $\begin{array}{l}\text { Nutrient } \\
\text { agar }\end{array}$ & NA \\
\hline 3 & Field 28 & $\begin{array}{l}\text { White/ } \\
\tan \end{array}$ & Unkown & Penicillin & $\begin{array}{l}\text { Nutrient } \\
\text { agar }\end{array}$ & NA \\
\hline 4 & Marquis A & Yellow & Unkown & Streptomycin & $\begin{array}{l}\text { Nutrient } \\
\text { agar }\end{array}$ & NA \\
\hline 5 & Marquis A & Yellow & Raised & NA & $\begin{array}{l}\text { Nutrient } \\
\text { agar }\end{array}$ & NA \\
\hline 6 & Arboretum & White & Spreading & NA & $\begin{array}{l}\text { TYES- } \\
\text { CR }\end{array}$ & $\begin{array}{l}\text { absorbed } \\
\mathrm{cr}\end{array}$ \\
\hline 7 & Marquis A & $\begin{array}{l}\text { Transl } \\
\text { yellow }\end{array}$ & Raised & NA & $\begin{array}{l}\text { Nutrient } \\
\text { agar }\end{array}$ & NA \\
\hline 8 & Marquis $\mathrm{A}$ & White & Gliding & NA & $\begin{array}{l}\text { Nutrient } \\
\text { agar }\end{array}$ & NA \\
\hline 9 & Marquis $\mathrm{A}$ & $\begin{array}{l}\text { Transl } \\
\text { yellow }\end{array}$ & Gliding & NA & $\begin{array}{l}\text { Nutrient } \\
\text { agar }\end{array}$ & NA \\
\hline 10 & Marquis A & Yellow & Flat & NA & $\begin{array}{l}\text { Nutrient } \\
\text { agar }\end{array}$ & NA \\
\hline 11 & Marquis A & $\begin{array}{l}\text { Transl } \\
\text { Yellow }\end{array}$ & $\begin{array}{l}\text { Highly motile } \\
\text { at } 4^{\circ} \mathrm{C}\end{array}$ & NA & $\begin{array}{l}\text { Nutrient } \\
\text { agar }\end{array}$ & NA \\
\hline 12 & Arboretum & $\begin{array}{l}\text { Clear/ } \\
\tan \end{array}$ & Mucoid & NA & TYES & $\begin{array}{l}\text { Intense } \\
\text { pink }\end{array}$ \\
\hline 13 & Arboretum & White & Flat & NA & TYES & Dark red \\
\hline
\end{tabular}




\begin{tabular}{|c|c|c|c|c|c|c|}
\hline 14 & Marquis A & Yellow & Raised & NA & $\begin{array}{l}\text { Nutrient } \\
\text { agar }\end{array}$ & NA \\
\hline 15 & Arboretum & Clear/tan & $\begin{array}{l}\text { Small } \\
\text { mucoid }\end{array}$ & NA & TYES & Light pink \\
\hline 16 & Marquis $A$ & $\begin{array}{l}\text { Clear/ } \\
\text { tan }\end{array}$ & Mucoid & NA & TYES & NA \\
\hline 17 & Marquis A & $\begin{array}{l}\text { Transl } \\
\text { yellow }\end{array}$ & Mucoid & NA & TYES & NA \\
\hline 18 & Arboretum & $\begin{array}{l}\text { Clear/ } \\
\tan \end{array}$ & Gliding & NA & TYES & Abs cr \\
\hline 19 & Arboretum & White & Spreading & NA & TYES & Abs cr ring \\
\hline 20 & Marquis A & $\begin{array}{l}\text { Transl } \\
\text { yellow }\end{array}$ & $\begin{array}{l}\text { Raised, } \\
\text { wrinkled }\end{array}$ & NA & $\begin{array}{l}1 / 2 \\
N A+A C\end{array}$ & NA \\
\hline 21 & Marquis A & $\begin{array}{l}\text { Transl } \\
\text { yellow }\end{array}$ & Small colony & NA & $\begin{array}{l}1 / 2 \mathrm{NA} \\
+\mathrm{AC}\end{array}$ & NA \\
\hline 22 & Marquis A & $\begin{array}{l}\text { Transl } \\
\text { yellow }\end{array}$ & Small colony & Griseofulvin & ISP-6 & NA \\
\hline 23 & Marquis A & $\begin{array}{l}\text { Yellow/ } \\
\text { orange }\end{array}$ & raised & Griseofulvin & ISP-6 & NA \\
\hline 24 & Marquis A & $\begin{array}{l}\text { White/ } \\
\text { grey }\end{array}$ & Large colony & Griseofulvin & ISP-6 & NA \\
\hline 25 & Marquis A & $\begin{array}{l}\text { Transl } \\
\text { yellow }\end{array}$ & Wrinkled & Griseofulvin & ISP-6 & NA \\
\hline
\end{tabular}

Figure 4. Phylip Maximum Likelihood Tree. 


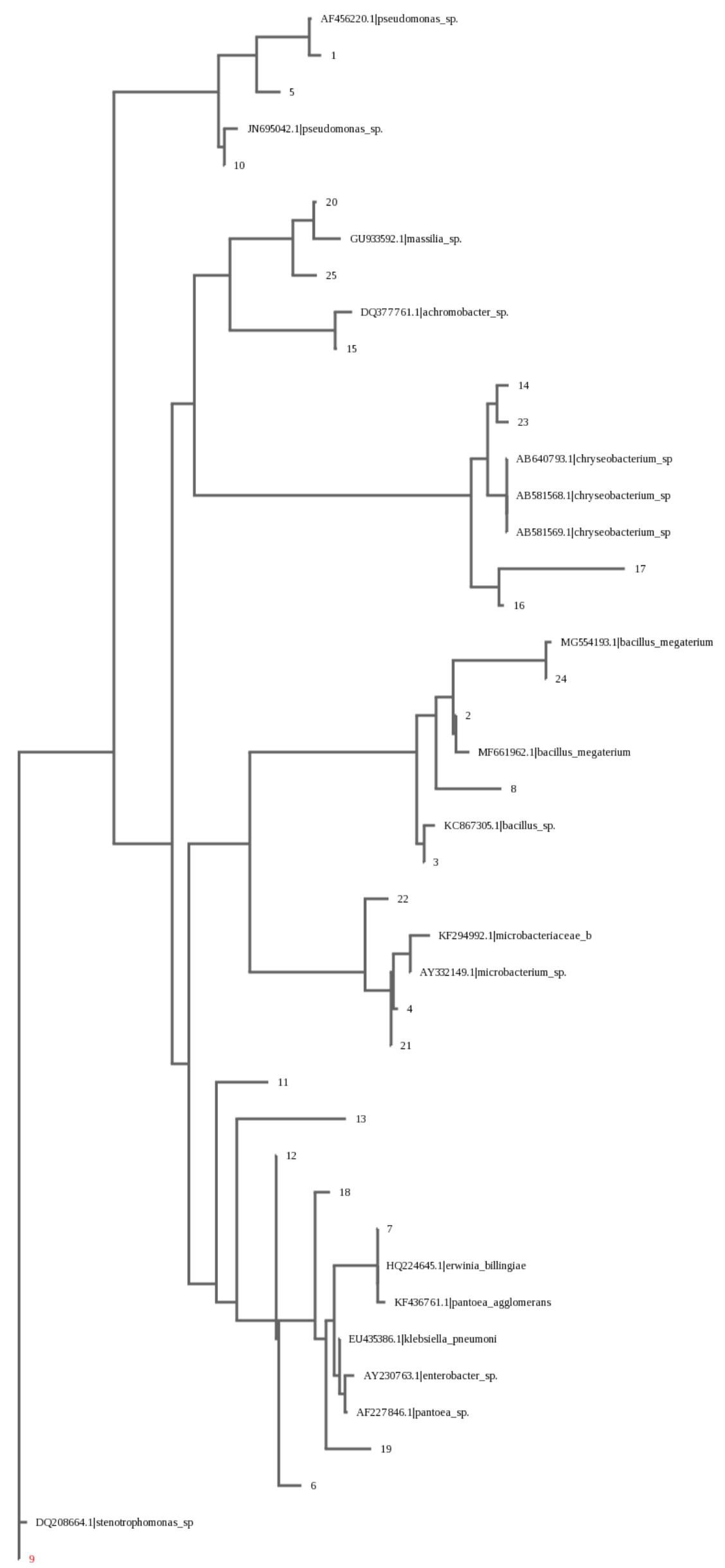


Table 3 Prokaryote Identification using EZBioCloud 16S-Based ID

\begin{tabular}{|c|c|c|c|c|}
\hline Name & Top-hit taxon & Top-hit strain & $\begin{array}{l}\text { Similarity } \\
(\%)\end{array}$ & $\begin{array}{l}\text { Completeness } \\
\text { (\%) }\end{array}$ \\
\hline Sample 1 & Pseudomonas mucoides & P154a & 84.46 & 52.2 \\
\hline Sample 2 & Priestia qingshengii & G19 & 71.85 & 66.6 \\
\hline Sample 3 & $N U Q E_{-} S$ & AFS041167 & 83.45 & 52.8 \\
\hline Sample 4 & Luethyella okanaganae & LBG B4405 & 82.3 & 51.7 \\
\hline Sample 5 & $\begin{array}{l}\text { Pseudomonas } \\
\text { neuropathica }\end{array}$ & P155 & 83.05 & 52.2 \\
\hline Sample 6 & Enterobacter huaxiensis & 90008 & 80.75 & 53.3 \\
\hline Sample 7 & Pantoea deleyi & LMG 24200 & 86.08 & 52.6 \\
\hline & & Marseille- & & \\
\hline Sample 8 & Bacillus mediterraneensis & P2366 & 82.08 & 55.2 \\
\hline Sample 9 & Xanthomonas maliensis & M97 & 81.03 & 53.5 \\
\hline Sample 10 & Pseudomonas bijieensis & L22-9 & 70.53 & 70.4 \\
\hline Sample 11 & Erwinia billingiae & CIP 106121 & 85.53 & 27.8 \\
\hline Sample 12 & CP009454_s & ND04 & 72.2 & 70.3 \\
\hline Sample 13 & Klebsiella huaxiensis & WCHKI090001 & 90.4 & 52.4 \\
\hline Sample 14 & Chryseobacterium tructae & $1084-08$ & 81.03 & 55.4 \\
\hline Sample 15 & Achromobacter deleyi & LMG 3458 & 82.94 & 52.5 \\
\hline Sample 16 & $\begin{array}{l}\text { Chryseobacterium } \\
\text { vietnamense }\end{array}$ & GIMN1.005 & 78.87 & 53.7 \\
\hline Sample 17 & $\begin{array}{l}\text { Chryseobacterium } \\
\text { aurantiacum }\end{array}$ & F30 & 59.61 & 78.2 \\
\hline Sample 18 & Pantoea dispersa & LMG 2603 & 83.51 & 53.6 \\
\hline Sample 19 & Pantoea eucrina & LMG 2781 & 85.74 & 52.4 \\
\hline Sample 20 & GU563773_s & Bmc86 & 72.27 & 65.2 \\
\hline Sample 21 & Luethyella okanaganae & LBG B4405 & 62.85 & 92.3 \\
\hline Sample 22 & Microbacterium binotii & CIP 101303 & 63.59 & 92.5 \\
\hline Sample 23 & $\begin{array}{l}\text { Chryseobacterium } \\
\text { phosphatilyticum }\end{array}$ & ISE14 & 80.7 & 55.9 \\
\hline Sample 24 & Priestia megaterium & NBRC 15308 & 82.65 & 53.7 \\
\hline Sample 25 & Massilia flava & Y9 & 80.44 & 52.4 \\
\hline
\end{tabular}

Differential Abundance Analysis in DESeq2

There were 223 virus, archaea, or bacterial genera that were differentially abundant between the Datura rootzone samples and the Fallow samples from Marquis Field near the compost pile. There were 140 species that were associated with the Datura rootzone and 83 species that were associated with the fallow samples, based on adjusted pvalue $<0.001$. Of those, 124 genera were also significant based on the log fold change cutoff of $1.33,34$ were associated with the Datura rootzone, and the remaining genera were associated with the fallow samples The results are summarized in Table 4 and visualized in Figure 5.

Table 4 . The 34 genera with both high negative $\log$ fold change values, and significant adjusted $p$-values $(p<0.001)$ representing those associated with the Datura rootzone are shown. 


\begin{tabular}{|c|c|c|c|}
\hline OTUID & Genus & log2FoldChange & p-adj. \\
\hline OTU149 & Cavemovirus & -8.74069944 & $1.46 \mathrm{E}-11$ \\
\hline OTU494 & Pantoea & -3.382739635 & $5.9 \mathrm{E}-18$ \\
\hline OTU168 & Chryseobacterium & -2.894493584 & 0.000172 \\
\hline OTU332 & Janthinobacterium & -2.396801275 & $5.53 \mathrm{E}-06$ \\
\hline OTU307 & Herbaspirillum & -2.385130454 & $2.86 \mathrm{E}-08$ \\
\hline OTU505 & Pedobacter & -2.384332893 & $6 \mathrm{E}-05$ \\
\hline OTU247 & Erwinia & -2.324319999 & $3.84 \mathrm{E}-35$ \\
\hline OTU738 & unclassified (derived from Flavobacteriaceae) & -2.299754251 & 0.00078 \\
\hline OTU273 & Gemmata & -2.299386567 & $3.76 \mathrm{E}-19$ \\
\hline OTU308 & Herminiimonas & -2.1461305 & 2.3E-05 \\
\hline OTU693 & Variovorax & -2.033564378 & $1.25 \mathrm{E}-07$ \\
\hline OTU759 & unclassified (derived from Verrucomicrobia subdivision 3) & -1.966909323 & $5.46 \mathrm{E}-18$ \\
\hline OTU169 & Chthoniobacter & -1.924054848 & $5.19 \mathrm{E}-11$ \\
\hline OTU440 & Mucilaginibacter & -1.920113231 & $6.47 \mathrm{E}-05$ \\
\hline OTU263 & Fluoribacter & -1.819462381 & 0.006919 \\
\hline OTU569 & Riemerella & -1.783874932 & 0.008796 \\
\hline OTU488 & Oxalobacter & -1.775720869 & $1.2 \mathrm{E}-06$ \\
\hline OTU339 & Klebsiella & -1.680008901 & 0.00538 \\
\hline OTU277 & Geodermatophilus & -1.67277933 & 0.000157 \\
\hline OTU463 & Nitrospira & -1.638446217 & 0.000467 \\
\hline OTU262 & Flavobacterium & -1.633855026 & 0.007125 \\
\hline OTU156 & Chitinophaga & -1.588995265 & 0.000676 \\
\hline OTU696 & Verrucomicrobium & -1.523905486 & 3.61E-05 \\
\hline OTU234 & Dyadobacter & -1.505907596 & 0.005228 \\
\hline OTU480 & Opitutus & -1.500266429 & $5.62 \mathrm{E}-05$ \\
\hline OTU598 & Serratia & -1.489698167 & $2.49 \mathrm{E}-10$ \\
\hline OTU421 & Methylobacterium & -1.448043272 & $2.59 \mathrm{E}-12$ \\
\hline OTU136 & Candidatus Solibacter & -1.428079685 & $1.1 \mathrm{E}-15$ \\
\hline OTU624 & Stigmatella & -1.392596868 & $6.52 \mathrm{E}-07$ \\
\hline OTU751 & unclassified (derived from Proteobacteria) & -1.373840383 & 0.003172 \\
\hline OTU180 & Conexibacter & -1.373047754 & 0.000188 \\
\hline OTU651 & Terriglobus & -1.362530369 & $6.27 \mathrm{E}-16$ \\
\hline OTU532 & Polaromonas & -1.340071098 & $9.35 \mathrm{E}-06$ \\
\hline OTU727 & unclassified (derived from Candidatus Poribacteria) & -1.330571533 & 0.00172 \\
\hline
\end{tabular}

Among the more notable results were differentially abundant bacteria in the Datura rootzone including Flavobacterium, Chryseobacterium, and unclassified members of the Flavobacteriaceae. Chitinophaga, Mucilaginibacter, and Pedobacter of the closely related Sphingobacteriaceae were also differentially associated with the Datura rootzone. These genera are associated with degradation of polysaccharides or chitin. There was also evidence that Verrucomicrobium that are typically associated with Flavobacterium as well as unclassified Verrucomicrobium were significantly associated with the Datura rootzone. These genera are known for hydrolysis of xylans. Dyadobacter of the Cytophagaceae, which has polysaccharide and amino acid degrading functions, was present in differentially large quantities; 
this species lives in glaciers and was formerly classified as a member Flavobacteriales. Stigmatella, which is responsible for breaking down insoluble debris, was also significantly associated with the Datura rootzone.

Herbasperillum and Oxalobacter from the Oxalobacteriaceae were differentially abundant, along with Janthinobacterium, which is produces violacein, an antifungal, antiprotist, antibacterial, and anti-tumor compound [30]. Interestingly, sequences from the plant pathogen Erwinia were also present in significantly high numbers, as well as the poultry disease Riemerella, and the infectious Serratia which is consistent with the animal bedding that is spread from the compost pile to all of the adjacent fields. Variovorax is involved in disrupting quorum sensing and has swarming motility, and was differentially abundant in the Datura rootzone. Bradyrhizobium and Azorhizobium abundance in the Datura rootzone was significant based on adjusted $p$-value $(\mathrm{p}<0.01)$ but not based on log fold change values.

Figure 5. Differential Abundance Analysis in R shows that there were a large number of taxa that were differentially expressed in the fallow (positive log fold change) versus Datura (negative log fold change) samples.

DESeq2 results for Virus, Bacteria, \& Archaea

\section{Differential abundance: Fallow vs. Datura inoxia}

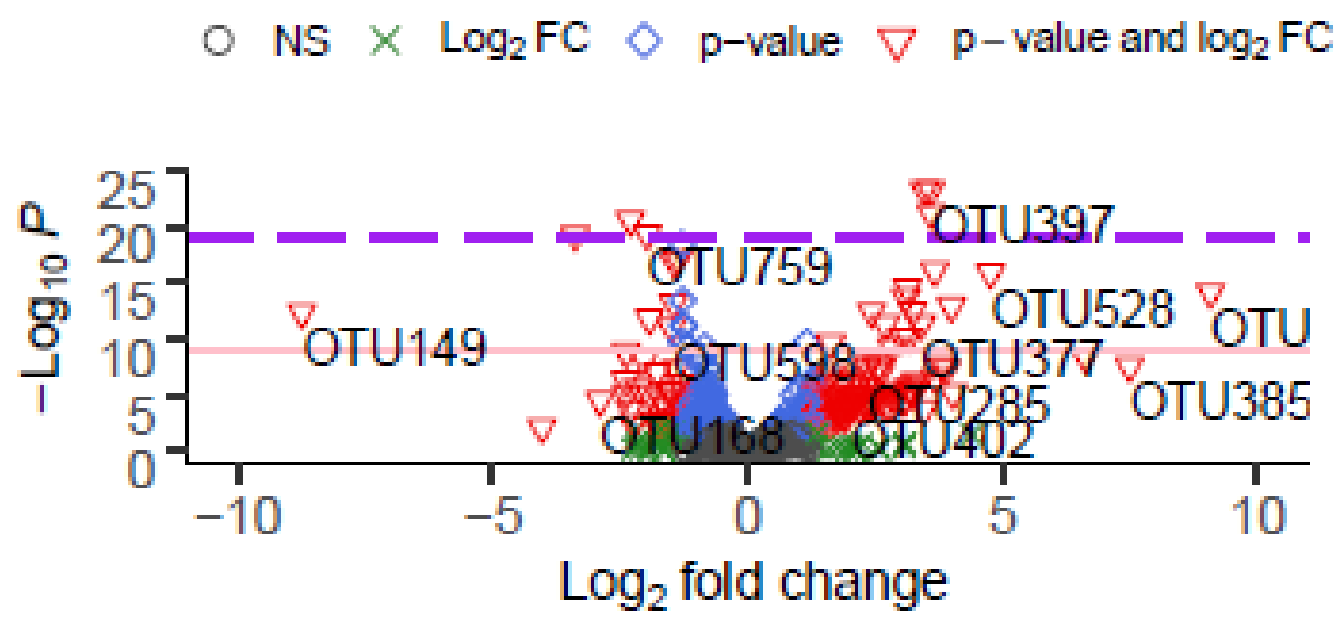

FC cutoff, 1.333 ; p-value cutoff, 0.01

Microbial barcoding and essential oil trial

The Phylip Maximum Likelihood phylogenetic tree is shown Figure 4. The results indicated that there are four main clusters of bacteria that were isolated. The first cluster includes isolates related to Pseudomonas sp. The second cluster consists of two groupings; one group is related to Massilia sp. and Achromobacter sp., while the second group is related to Chryseobacterium sp. The third cluster consisted of Bacillus sp. and Microbacteriaceae. Interestingly, Priestia megaterium is used as an herbicide in organic production [44]. The fourth cluster is made up of mostly pathogenic species such as Erwinia, Pantoea, and Klebsiella. Most of the pathogenic strains were associated with the Arboretum samples. Stenotrophomonas was used as the outgroup. As shown in Figure 4 and Table 3, there were four Chryseobacterium isolates that were detected with barcoding, which were not detected with 16S NGS metabarcoding. The ascertainment of the Achromobacter isolate agrees with NGS results. The Xanthomonas isolation agrees with the WGS results, since Xanthomonadaceae was the top family detected in the WGS samples for the Datura inoxia rootzone.

The main findings of the essential oil trials indicate that Pseudomonas mucoides was not susceptible to penicillin, and not susceptible to lemon balm essential oils. In 
retrospect, since $P$. mucoides is a gram-negative bacterium it would not be expected to be susceptible to penicillin. Bacillus pseudomycoides was resistant to penicillin but grew even more with lemon balm and tea tree essential oils. It is interesting because B. pseudomycoides was tolerant of high alcohol content, possibly since it is a lactic acid fermenter. Priestia qingshengii was not susceptible to penicillin and grew less when lemon balm or tea tree essential oils were added. Leuthyella okanaganae was resistant to streptomycin; however, growth was controlled significantly when lemon balm or tea tree essential oils were added and the least growth occurred when the tea tree treatment was used.

Figure 6. The interaction plot for the Bacteria and Treatment variables of the essential oil trial are shown. Interestingly, there was an interaction between the essential oil used and the bacterial accession it was tested against.

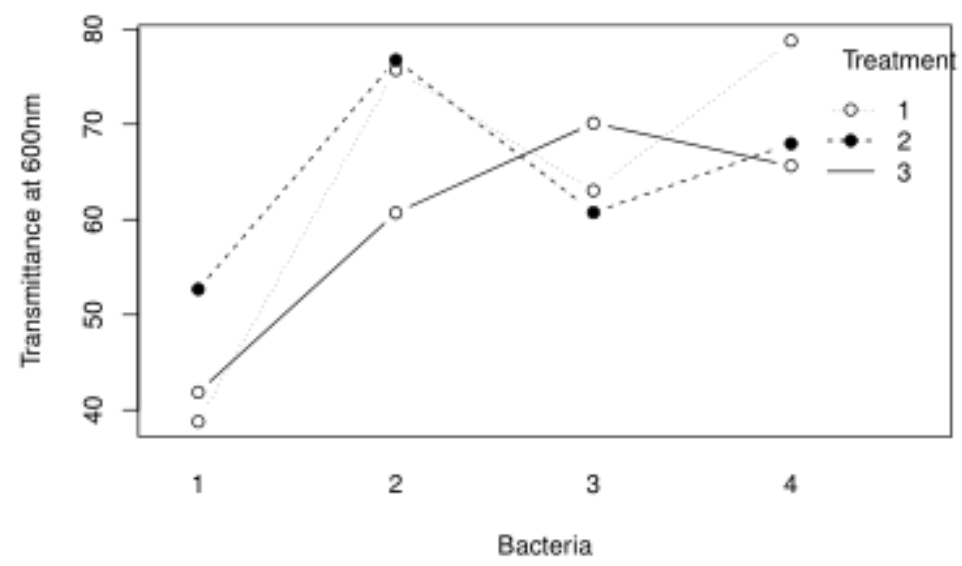

It is interesting because Luna et al 2007 found that several Bacillus pseudomycoides strains were susceptible to beta-lactams, however the strain ascertained in this study was resistant to penicillin [31]. Bacillus pseudomycoides is related to Bacillus anthracis and Bacillus cereus; these species are notorious for being antibiotic resistant but B. pseudomycoides and $B$. cereus are particularly resistant to clindamycin [31].

Pseudomonas is also notoriously resistant to antibiotics, although its inclusion here was somewhat serendipitous since it was not selected with an antibiotic that would be typically used to combat gram negative bacteria. It was also found that tea tree and lemon balm oils were ineffective against the $P$. mycoides isolate.

\section{Discussion}

Cytophagaceae are known to degrade polysaccharides [32]. Pedobacter inoculation has been shown to significantly increase the antioxidant content of strawberries [33]. Closely related species are the Flavobacterium, which are famous for producing flavonoids including quinones and they play a role in disease suppression in soils [34]. Flavonoid and alkaloid production gene ontologies are closely related [35, 36]. Rhizoctonia solani is suppressed by Flavobacterium [37]. This is believed to be related to its chitinase activity which is also of interest for energy production from biomass [38].

As Fadiji 2020 pointed out, plant protective endophytes stimulate plant secondary metabolite production while inducing plant resistance to pathogens [39]. In addition, endophytic fungi produce strong antioxidants. In Taxus cuspidata paclitaxel production was elevated, and in Euphorbia pekinensis there was elevated terpene production in response to the Fusarium E5 elicitor; these are two examples from the article. Some examples of protection provided by the endophytes include antibiotics, competition for resources with pathogens, and lytic enzymes such as chitinases that break down fungi and plant cell walls.

The chitinase activity provides a possible answer to how endophytes and their products are transported into the plant. Furthermore, xylanase and other cell wall degrading 
enzymes such as chitinases and cellobio-hydrolase were detected in the metagenome of several endophytes [17]. These lytic enzymes have the dual function of giving microbes access to plant cells, ducts, and intercellular spaces for colonization and transport, and the enzymes can also break down fungi cell walls to assist the plant host while fighting off infections.

Our preliminary evidence shows that the functional hits for Chalcone synthase in the microbes of the Datura rootzone are differentially abundant in contrast to the fallow sample. Chalcone synthase is involved in antioxidant production in bacteria and plants, and is homologous with polyketide synthase, which is part of the alkaloid production pathway in plants [40,41]. There appears to be synergy between the plant and microbe functions in terms of secondary metabolite production in the rootzone.

\section{Genetic Engineering and the Flavonoid Biosynthesis Pathway}

Another way to look at this is that microbes may be engineered to produce the plant medicinal compounds directly [42]. Chemical synthesis of aromatic compounds uses benzene, toluene, and xylene as starting materials; these materials are derived from petroleum. Microorganisms are a renewable source of plant-derived secondary compounds. These compounds include phenolic acids, flavonoids, stilbenoids, coumarin, and conjugates of the same. A pathway of interest for the engineering of microbes for the production of plant metabolites, which occurs in nature, is the pathway by which tyrosine is converted to p-coumaric acid by tyrosine ammonia lyase and later converted to quercetin.

Yonekura et al 2019 shed light on the flavonoid biosynthesis pathway [35]. In the plant kingdom, flavonoids are widely distributed in all subclasses, except for hornworts. This suggests that chalcone synthase, the first enzyme in the pathway, was evolved many times in parallel. If the enzyme was universal, then it must have been lost by many species along the way, since not all species within the subclasses have it [35]. The first two enzymes on the biosynthetic pathway are chalcone synthase and chalcone isomerase, which are believed to also have evolved first from a common ancestor involved in lipid metabolism.

There are 9,000 flavonoid compounds defined strictly as:

1. Structural derivatives of phenyl-substituted propylbenzenes with a $\mathrm{C} 15$ backbone,

2. Phenyl-substituted propylbenzene derivatives with a C16 skeleton, or

3. Phenyl-substituted propylbenzenes condensed with C6-C3 lignan precursors to form flavonolignans.

More broadly, we can also include chalcones and dihydrochalcones, anthocyanins, and aurones [35]. It is believed that defense against UV radiation, and plant hormone regulation were the first flavonoid functions. Flavonoid accumulation could help with harsh field conditions in Datura. They are structurally related to carotenoids, which are part of the accessory light harvesting complex in plants. Interestingly, UV-B radiation of mosses increased flavonoid content, which supports this notion [35].

Chalcone synthase (CHS) is derived from beta-ketoacyl ACP synthase. It is in the Type III polyketide synthase (PKS) gene family. CHS may have evolved before chalcone isomerase (CHI), since CHS catalyzes the first step and the step catalyzed by $\mathrm{CHI}$ may happen spontaneously [35]. P-coumaroyl CoA is transformed to naringenin chalcone by $\mathrm{CHS}$, then to naringenin by CHI. Arabidopsis has four Type III PKS genes and one functional CHS. 
CHS is promiscuous in the sense that it can use many substrates, although it cannot function on bulky substrates [35]. Flavobacterium johnsoniae has a chalcone synthase gene according to the Uniprot database [43], which may be working synergistically with Datura to increase flavonoid content in the plant, supply acyl compounds to the plant for downstream production for plant secondary metabolites, or have a modified function that is specific to its plant host. It is worth mentioning that Datura, Streptomyces, and Agrobacterium also have this gene. In vitro, CHS from Huperzia serrata can produce other polyketides besides flavonoids, like aromatic tricyclic pyridoisoindoles [35].

Evolutionarily, CHS on the flavonoid biosynthetic pathway may have paved the way for biosynthesis of medicinal secondary metabolites by land plants. It expanded the chemical variety of the metabolites plants produced over time [35]. Type III PKS can also synthesize oxidosqualane and terpenes in other specialized metabolisms, for example. It is not known why plants and their endophytes have evolved this important set of enzymes, but it warrants further investigation.

There was strong evidence to support the hypothesis that synergistic production of plant derived secondary metabolites by microbes occurred in the Datura rootzone, as well as the ability for the compounds to enter plant cells. Many medicinal plants produce economically important compounds, and synthetic production is not possible because the pathways are poorly understood. Further elucidating the interaction between microbes and wild plants could also shed light on resilience mechanisms, which could be used for crop improvement. For example, it has been shown that beneficial microbes prime plant defenses, giving a competitive advantage to organic farming [45]. Furthermore, understanding how these plants adapt to their environment will empower weed control efforts, including biocontrol.

Author Contributions: Conceptualization, S.S.; formal analysis, S.S.; investigation, SS., K.P., A.B.; writing-original draft preparation, S.S.; writing-review and editing, K.P., A.B.; All authors have read and agreed to the published version of the manuscript.

Funding: This work was supported by training and resources from the NSF IUSE grant, NSF DUE1821657 awarded to Cold Spring Harbor Laboratory and James Madison University, in part to support Community College faculty. This material is based upon work supported by the National Science Foundation under Grant Nos. DBI-0735191, DBI-1265383, and DBI-1743442 which supported iPlant Collaborative and DNA Subway development.

Institutional Review Board Statement: Not applicable.

Informed Consent Statement: Not applicable.

Data Availability Statement: WGS profiles, metadata and genomic data are freely available on MGRAST under public project mgp98747. DNA Subway Purple Line metabarcoding results are available on Cyverse under public project 7102. Raw fastq files are available at: https://de.cyverse.org/data/ds/iplant/home/shared/iplant_DNA_subway/metabarcod-

ing_data/210611_M00970/St_Claire. DNA Subway Blue line barcoding results and trace files are available under public project 238876 on Cyverse.

Acknowledgments: Thank you to Maryam Saraylou for advice on cultivation dependent methods. Thank you to Daila Melendez, Rosealie Vicuna, and Karu Smith for assistance with plant material extraction. Thank you to Bruce Nash for materials. Thank you to Jay R. Reichman for technical writing assistance.

Conflicts of Interest: The authors declare no conflict of interest. The funders had no role in the design of the study; in the collection, analyses, or interpretation of data; in the writing of the manuscript, or in the decision to publish the results.

\section{References}

1. Soni, Priyanka et al. "Pharmacological properties of Datura stramonium L. as a potential medicinal tree: an overview." Asian Pacific journal of tropical biomedicine vol. 2,12 (2012): 1002-8. doi:10.1016/S2221-1691(13)60014-3.

2. Han, Xiao-lin et al. Zhong yao cai $=$ Zhongyaocai = Journal of Chinese medicinal materials vol. 38,8 (2015): 1646-8.

3. Lemos Ari S. O., Florêncio Jônatas R., Pinto Nícolas C. C., Campos Lara M., Silva Thiago P., Grazul Richard M., Pinto Priscila F., Tavares Guilherme D., Scio Elita, Apolônio Ana Carolina M., Melo Rossana C. N., Fabri Rodrigo L. Antifungal Activity of the 
Natural Coumarin Scopoletin Against Planktonic Cells and Biofilms From a Multidrug-Resistant Candida tropicalis Strain. Frontiers in Microbiology 11 (2020), page 1525. https://www.frontiersin.org/article/10.3389/fmicb.2020.01525 Accessed 9/12/2021.

4. SIKLÓS, BULCSU. “DATURA RITUALS IN THE VAJRAMAHABHAIRAVA-TANTRA.” Acta Orientalia Academiae Scientiarum Hungaricae, vol. 47, no. 3, 1994, pp. 409-416. JSTOR, www.jstor.org/stable/23658487. Accessed 18 July 2021.

5. Rajewski, A., Carter-House, D., Stajich, J. et al. Datura genome reveals duplications of psychoactive alkaloid biosynthetic genes and high mutation rate following tissue culture. BMC Genomics 22, 201 (2021). https://doi.org/10.1186/s12864-021-07489-2

6. David W. Robinson, Kelly Brown, Moira McMenemy, Lynn Dennany, Matthew J. Baker, Pamela Allan, Caroline Cartwright, Julienne Bernard, Fraser Sturt, Elena Kotoula, Christopher Jazwa, Kristina M. Gill, Patrick R Quinney, Thomas Ash, Clare Bedford, Devlin Gandy, Matthew Armstrong, James Miles, David Haviland. Datura quids at Pinwheel Cave, California, provide unambiguous confirmation of the ingestion of hallucinogens at a rock art site. Proceedings of the National Academy of Sciences Dec 2020, 117 (49) 31026-31037; DOI: 10.1073/pnas.2014529117

7. Simon Irmer, Nora Podzun, Dorothee Langel, Franziska Heidemann, Elisabeth Kaltenegger, Brigitte Schemmerling, ChristophMartin Geilfus, Christian Zörb, Dietrich Ober. Nodulation and alkaloid biosynthesis in Crotalaria. Proceedings of the National Academy of Sciences Mar 2015, 112 (13) 4164-4169; DOI: 10.1073/pnas.1423457112

8. Xia, Yun, Dong, Minghua, Yu, Lei, Kong, Lingdong, Seviour, Robert, and Kong, Yunhong. "Compositional and Functional Profiling of the Rhizosphere Microbiomes of the Invasive Weed Ageratina Adenophora and Native Plants." PeerJ (San Francisco, CA) 9 (2021): E10844. Web.

9. Sang, M K, and K D Kim. “The volatile-producing Flavobacterium johnsoniae strain GSE09 shows biocontrol activity against Phytophthora capsici in pepper." Journal of applied microbiology vol. 113,2 (2012): 383-98. doi:10.1111/j.1365-2672.2012.05330.x

10. VÍCTOR J. CARRIÓN, JUAN PEREZ-JARAMILLO, VIVIANE CORDOVEZ, VITTORIO TRACANNA, MATTIAS DE HOLLANDER, DANIEL RUIZ-BUCK, LUCAS W. MENDES, WILFRED F.J. VAN IJCKEN, RUTH GOMEZ-EXPOSITO, SOMAYAH S. ELSAYED, PRARTHANA MOHANRAJU, ADINI ARIFAH, JOHN VAN DER OOST, JOSEPH N. PAULSON, RODRIGO MENDES, GILLES P. VAN WEZEL, MARNIX H. MEDEMA, JOS M. RAAIJMAKERS. Pathogen-induced activation of diseasesuppressive functions in the endophytic root microbiome SCIENCE01 NOV $2019: 606-612$.

11. Lucke Miriam, Correa Mario Gabriel, Levy Asaf. The Role of Secretion Systems, Effectors, and Secondary Metabolites of Beneficial Rhizobacteria in Interactions With Plants and Microbes. Frontiers in Plant Science 11. 2020. 1718. https://www.frontiersin.org/article/10.3389/fpls.2020.589416

12. del Barrio-Duque A, Ley J, Samad A, Antonielli L, Sessitsch A and Compant S (2019) Beneficial Endophytic Bacteria-Serendipita indica Interaction for Crop Enhancement and Resistance to Phytopathogens. Front. Microbiol. 10:2888. doi: 10.3389/fmicb.2019.02888

13. Morris JS and Facchini PJ (2019) Molecular Origins of Functional Diversity in Benzylisoquinoline Alkaloid Methyltransferases. Front. Plant Sci. 10:1058. doi: 10.3389/fpls.2019.01058

14. Bañuelos-Vazquez Luis Alfredo, Cazares Daniel, Rodríguez Susana, Cervantes-De la Luz Laura, Sánchez-López Rosana, Castellani Lucas G., Tejerizo Gonzalo Torres, Brom Susana. Transfer of the Symbiotic Plasmid of Rhizobium etli CFN42 to Endophytic Bacteria Inside Nodules. Frontiers in Microbiology 11. 2020. Page 1752. https://www.frontiersin.org/article/10.3389/fmicb.2020.01752

15. Wu, Wei et al. "Beneficial Relationships Between Endophytic Bacteria and Medicinal Plants." Frontiers in plant science vol. 12 646146. 22 Apr. 2021, doi:10.3389/fpls.2021.646146

16. Flores-Bustamante, Zoila R, Rivera-Orduña, Flor N, Martínez-Cárdenas, Anahí, and Flores-Cotera, Luis B. "Microbial Paclitaxel: Advances and Perspectives." Journal of Antibiotics 63.8 (2010): 460-67. Web.

17. Oukala, N.; Aissat, K.; Pastor, V. Bacterial Endophytes: The Hidden Actor in Plant Immune Responses against Biotic Stress. Plants 2021, 10, 1012. https://doi.org/10.3390/plants10051012

18. Soil Survey Staff, Natural Resources Conservation Service, United States Department of Agriculture. Web Soil Survey. Available online at the following link: http://websoilsurvey.sc.egov.usda.gov/.

19. Chen, Yuxin et al. "SOAPnuke: a MapReduce acceleration-supported software for integrated quality control and preprocessing of high-throughput sequencing data." GigaScience vol. 7,1 (2018): 1-6. doi:10.1093/gigascience/gix120

20. Meyer, F., Paarmann, D., D'Souza, M. et al. The metagenomics RAST server - a public resource for the automatic phylogenetic and functional analysis of metagenomes. BMC Bioinformatics 9, 386 (2008). https://doi.org/10.1186/1471-2105-9-386

21. Love MI, Huber W, Anders S (2014). "Moderated estimation of fold change and dispersion for RNA-seq data with DESeq2." Genome Biology, 15, 550. doi: 10.1186/s13059-014-0550-8.

22. Parks, Donovan H et al. "STAMP: statistical analysis of taxonomic and functional profiles." Bioinformatics (Oxford, England) vol. 30,21 (2014): 3123-4. doi:10.1093/bioinformatics/btu494

23. Uwe Hilgert, Sheldon McKay, Mohammed Khalfan, Jason Williams, Cornel Ghiban, and David Micklos. 2014. DNA Subway: Making Genome Analysis Egalitarian. In Proceedings of the 2014 Annual Conference on Extreme Science and Engineering Discovery Environment. Association for Computing Machinery, New York, NY, USA, Article 70, 1-3. DOI:https://doi.org/10.1145/2616498.2616575

24. J. Gregory Caporaso, Christian L. Lauber, William A. Walters, Donna Berg-Lyons, Catherine A. Lozupone, Peter J. Turnbaugh, Noah Fierer, Rob Knight. Global patterns of $16 \mathrm{~S}$ rRNA diversity at a depth of millions of sequences per sample. Proceedings of the National Academy of Sciences Mar 2011, 108 (Supplement 1) 4516-4522; DOI: 10.1073/pnas.1000080107

25. Caporaso, J., Lauber, C., Walters, W. et al. Ultra-high-throughput microbial community analysis on the Illumina HiSeq and MiSeq platforms. ISME J 6, 1621-1624 (2012). https://doi.org/10.1038/ismej.2012.8 
26. Savanah St. Clair, Maryam Saraylou, Elnura Maine 2019. Introduction to Soil Science Lab Manual. protocols.io https://dx.doi.org/10.17504/protocols.io.5jzg4p6

27. Yoon, S. H., Ha, S. M., Kwon, S., Lim, J., Kim, Y., Seo, H. and Chun, J. (2017). Introducing EzBioCloud: A taxonomically united database of 16S rRNA and whole genome assemblies. Int J Syst Evol Microbiol. 67:1613-1617.

28. Eudes, Aymerick et al. "Exploiting members of the BAHD acyltransferase family to synthesize multiple hydroxycinnamate and benzoate conjugates in yeast." Microbial cell factories vol. 15,1 198. 21 Nov. 2016, doi:10.1186/s12934-016-0593-5

29. Jendresen, Christian Bille et al. "Highly Active and Specific Tyrosine Ammonia-Lyases from Diverse Origins Enable Enhanced Production of Aromatic Compounds in Bacteria and Saccharomyces cerevisiae." Applied and environmental microbiology vol. 81,13 (2015): 4458-76. doi:10.1128/AEM.00405-15

30. Ines Friedrich, Jacqueline Hollensteiner, Dominik Schneider, Anja Poehlein, Robert Hertel, Rolf Daniel, First Complete Genome Sequences of Janthinobacterium lividum EIF1 and EIF2 and Their Comparative Genome Analysis, Genome Biology and Evolution, Volume 12, Issue 10, October 2020, Pages 1782-1788, https://doi.org/10.1093/gbe/evaa148

31. Luna, Vicki A et al. "Susceptibility of Bacillus anthracis, Bacillus cereus, Bacillus mycoides, Bacillus pseudomycoides and Bacillus thuringiensis to 24 antimicrobials using Sensititre automated microbroth dilution and Etest agar gradient diffusion methods." The Journal of antimicrobial chemotherapy vol. 60,3 (2007): 555-67. doi:10.1093/jac/dkm213

32. Baek K, Choi A. Draft genome sequence of the polysaccharide degrading bacterium Cytophagaceae strain SJW1-29. Korean J. Microbiol. 2020; 56:422-425. https://doi.org/10.7845/kjm.2020.0112

33. Maria Morais et al. Comparative study of plant growth-promoting bacteria on the physiology, growth, and fruit quality of strawberry. Journal of the Science of Food and Agriculture 99(12). May 2019.

34. Kolton et al. Microbial Models from Environmental to Industrial Sustainabilty. Ch 9: The Flavobacterium genus in the Plant Holobiont: Ecological, Physiological, and Applicative Insights. 2016.

35. Yonekura-Sakakibara et al. The Origin and Evolution of Plant Flavonoid Metabolism. Frontiers in Plant Science. 2019.

36. Abe. Biosynthesis of medicinally important plant metabolites by unusual type III polyketide synthases. Journal of Natural Medicines. 2020.

37. Schlatter et al. Disease Suppressive Soils: New Insights from the Soil Microbiome. Phytopathology Review. June 2017.

38. Nordberg H, Cantor M, Dusheyko S, Hua S, Poliakov A, Shabalov I, Smirnova T, Grigoriev IV, Dubchak I. Nucleic Acids Res. 2014,42(1): D26-31. The genome portal of the Department of Energy Joint Genome Institute: 2014 updates.

39. Fadiji, Ayomide Emmanuel, and Olubukola Oluranti Babalola. “Elucidating Mechanisms of Endophytes Used in Plant Protection and Other Bioactivities With Multifunctional Prospects." Frontiers in bioengineering and biotechnology vol. 8 467. 15 May. 2020, doi:10.3389/fbioe. 2020.00467

40. Bedewitz et al. Tropinone synthesis via an atypical polyketide synthase and P450 mediated cyclization. Nature Communications. 2018.

41. Huang et al. Tropane alkaloids biosynthesis involves an unusual type III polyketide synthase and non-enzymatic condensation. Nature Communications. 2019.

42. Huccetogullari, D., Luo, Z.W. \& Lee, S.Y. Metabolic engineering of microorganisms for production of aromatic compounds. Microb Cell Fact 18, 41 (2019). https://doi.org/10.1186/s12934-019-1090-4

43. The UniProt Consortium. UniProt: the universal protein knowledgebase in 2021. Nucleic Acids Res. 49:D1 (2021)

44. Dara, S. K. (2021, April). Biopesticides and their role in integrated pest management. CAPCA Adviser, 48.

45. Krey Karol L., Nabity Paul D., Blubaugh Carmen K., Fu Zhen, Van Leuven James T., et al. Organic Farming Sharpens Plant Defenses in the Field. Frontiers in Sustainable Food Systems vol 4. 2020: 97 https://www.frontiersin.org/article/10.3389/fsufs.2020.00097

46. Clarence I. Kado and Brian Kelly. 2006. Transformation of Actinomycetes. Agrobacterium Protocols Volume 2. 395-402. 